\title{
On Positive Decaying Solutions of Quasilinear Ordinary Differential Equations with Singular Nonlinearities
}

\author{
By \\ Misako Motai and Hiroyuki Usami* \\ (Hiroshima University, Japan)
}

\begin{abstract}
We consider singular boundary value problems to quasilinear ordinary differential equations with singular nonlinearities. We give sufficient conditions for such problems to have a positive solution. Moreover, for a typical case we can show also the uniqueness of the solutions. solution.

Key Words and Phrases. Quasilinear ordinary differential equation, Positive

2000 Mathematics Subject Classification Numbers. Primary 34E10, Secondary $34 \mathrm{D} 05$.
\end{abstract}

\section{Introduction and main results}

Let us consider the following singular boundary value problem:

$$
\begin{gathered}
\left(\left|x^{\prime}\right|^{\alpha-1} x^{\prime}\right)^{\prime}=q(t) x^{-\beta}, \quad t \geq t_{0}, \\
\lim _{t \rightarrow \infty} x(t)=0 .
\end{gathered}
$$

For equation (1) we always assume that:

$\left(\mathrm{A}_{1}\right) \quad \alpha, \beta>0$ are constants;

$\left(\mathrm{A}_{2}\right) \quad q \in C\left(\left[t_{0}, \infty\right) ; \boldsymbol{R}\right)$, and $\operatorname{supp} q$, the support of $q(t)$, is unbounded;

$\left(\mathrm{A}_{3}\right) \quad q$ is integrable on $\left[t_{0}, \infty\right)$, and it satisfies $\int_{t}^{\infty} q(s) d s \geq 0, t \geq t_{0}$.

By assumption $\left(\mathrm{A}_{3}\right)$ we can introduce the auxiliary function

$$
Q(t)=\int_{t}^{\infty} q(s) d s \geq 0 \quad \text { for } t \geq t_{0} .
$$

Since $\operatorname{supp} q$ is unbounded, so is $\operatorname{supp} Q$. Note that $q(t)$ is allowed to change the sign near $+\infty$. In the paper $Q(t)$ will play an important role.

A function $x:\left[t_{0}, \infty\right) \rightarrow(0, \infty)$ is said to be a positive solution of (1) if $x$ and $\left|x^{\prime}\right|^{\alpha-1} x^{\prime}$ are of class $C^{1}\left[t_{0}, \infty\right)$ and satisfy equation $(1)$ on $\left[t_{0}, \infty\right)$. A positive solution of problem (1)-(2) is often called positive decaying solution of equation (1).

\footnotetext{
* Partly Supported by Grant-in-Aid for Scientific Research (C)-17540159, Japan Society for the Promotion of Science.
} 
It is known [1, 2] that singular differential equations such as (1) arise, for example, in the boundary layer theory of viscous fluids. When $\alpha=1$ and $q(t) \geq 0$, the study of this problem was initiated by $[1,3]$ as far as the authors are aware. In $[1,6]$ the existence of positive solutions of (1)-(2) with $\alpha=1$ was discussed. One of the authors obtained in [7] systematic results concerning asymptotic behavior of positive solutions to equation (1) with $\alpha=1$. The aim of the paper is to establish sufficient conditions for problem (1)-(2) to have at least one positive solution. More precisely, we intend not only to extend known results, for example, in $[1,6,7]$ to the general case where $\alpha>0$, but also to establish new existence theorems. In fact, one of our results presented here are new even though $\alpha=1$. Related results for the general case $\alpha>0$ are found in [4].

In the case of $q(t) \geq 0, t \geq t_{0}$, if either $\left(\mathrm{A}_{2}\right)$ or $\left(\mathrm{A}_{3}\right)$ is violated, then problem (1)-(2) never has positive solutions. Therefore it is natural to assume $\left(\mathrm{A}_{2}\right)$ and $\left(\mathrm{A}_{3}\right)$ in advance; see Remark 2 for details. Roughly speaking, problem (1)-(2) has a positive solution when $q(t)$ is sufficiently small near $+\infty$.

The main results of the paper are as follows:

Theorem 1. Let the integral conditions

$$
\begin{gathered}
\int^{\infty} Q(t)^{1 / \alpha} d t<\infty \\
\int^{\infty}\left(\int_{t}^{\infty} Q(s)^{(1+\alpha) / \alpha} d s\right)^{1 / \alpha} d t<\infty,
\end{gathered}
$$

and

$$
\int^{\infty}\left\{\int_{t}^{\infty}|q(s)|\left(\int_{s}^{\infty} Q(r)^{1 / \alpha} d r\right)^{-\alpha \beta /(\alpha+\beta)} d s\right\}^{1 / \alpha} d t<\infty
$$

hold. Then, problem (1)-(2) has a positive solution.

When $0<\beta<1$, we can obtain the results of other types, which may be more convenient to apply.

Theorem 2. Let $0<\beta<1$, and (3) and (4) hold. Suppose moreover that there is a nonincreasing function $\hat{q} \in C\left(\left[t_{0}, \infty\right) ;(0, \infty)\right)$ satisfying

$$
|q(t)| \leq \hat{q}(t), \quad t \geq t_{0},
$$

and

$$
\int^{\infty} \hat{q}(t)^{1 /(\alpha+1)} d t<\infty .
$$

Then, problem (1)-(2) has a positive solution. 
Theorem 3. Let $0<\beta<1$, and (3) and (4) hold. Suppose moreover that there is a constant $\delta \in(\beta, 1)$ satisfying

$$
\int^{\infty}\left(\int_{t}^{\infty}|q(s)|^{1 /(1-\delta)} d s\right)^{(1-\delta) /(\alpha+\delta)} d t<\infty .
$$

Then, problem (1)-(2) has a positive solution.

Remark 1. (i) When $\alpha=1$, Theorems 1 and 2 reduce to [6, Theorems 1 and 2], respectively.

(ii) Since, for each $p>0$, there are nonnegative and unbounded continuous functions belonging to $L^{p}\left[t_{0}, \infty\right)$, Theorems 2 and 3 are independent.

An immediate consequence of Theorem 2 (or Theorem 3) follows:

Corollary 4. Let $0<\beta<1$. If $q(t)=O\left(t^{-v}\right)$ as $t \rightarrow \infty$ for some $v>\alpha+1$, then problem (1)-(2) has a positive solution.

Remark 2. Suppose that $q(t)>0$ and problem (1)-(2) has a positive solution $x(t)$. Then we can show that $q(t)$ is integrable, and (3) and (4) hold. To see this we firstly notice that $x^{\prime}(t)<0$ and $\lim _{t \rightarrow \infty} x^{\prime}(t)=0$. Let us rewrite equation (1) as $-\left\{\left[-x^{\prime}(t)\right]^{\alpha}\right\}^{\prime} x(t)^{\beta}=q(t)$. Integrating on $[t, \tau]$, we obtain

$$
\left[-x^{\prime}(t)\right]^{\alpha} x(t)^{\beta} \geq \int_{t}^{\tau} q(s) d s, \quad \tau \geq t \geq t_{0},
$$

where integral by part and the decreasing nature of $x(t)$ are employed. Letting $\tau \rightarrow \infty$, we obtain $\left[-x^{\prime}(t)\right]^{\alpha} x(t)^{\beta} \geq \int_{t}^{\infty} q(s) d s$; that is $-x^{\prime}(t) x(t)^{\beta / \alpha} \geq Q(t)^{1 / \alpha}$. Hence one more integration gives $\int^{\infty} Q(t)^{1 / \alpha} d s<\infty$. Since $Q(t)$ is decreasing, we can obtain

$$
\begin{aligned}
\int_{t_{0}}^{\infty}\left(\int_{t}^{\infty} Q(s)^{(1+\alpha) / \alpha} d s\right)^{1 / \alpha} d t & \leq \int_{t_{0}}^{\infty} Q(t)^{1 / \alpha}\left(\int_{t}^{\infty} Q(s)^{1 / \alpha} d s\right)^{1 / \alpha} d t \\
& \leq\left(\int_{t_{0}}^{\infty} Q(t)^{1 / \alpha} d t\right)^{1 / \alpha+1}<\infty
\end{aligned}
$$

We do not know whether or not under conditions (3) and (4), without any additional conditions, problem (1)-(2) has a positive solutions. This is an unsettled problem.

Remark 3. Let $\liminf \operatorname{in}_{t \rightarrow \infty} t^{v} q(t)>0$ for some $v>0$. As is seen from Remark 1, if problem (1)-(2) has a positive solutions, then $v>\alpha+1$. This fact shows that the decaying order of $q(t)$ required in the assumption of Corollary 4 is the best possible in some sense. We conjecture that Corollary 4 is still valid even though $\beta \geq 1$. 
The paper is organized as follows. In Section 2 we give proofs of the main results. For this purpose we prepare an important lemma Lemma 5. At the end of Section 2 we give illustrative examples. In Section 3 we consider problem (1)-(2) in the restricted case where $q(t)=t^{-v}, v>0$. We then prove that, in this case, this problem has exactly one solution given explicitly if $v>\alpha+1$.

\section{Proofs of main results}

To establish our results we need the following lemma.

Lemma 5. Let integral conditions (3) and (4) hold. Then, for any constant $\ell>0$, equation (1) has a positive solution $x$ satisfying $x^{\prime}(t)<0, \quad t \geq t_{0}$, $\lim _{t \rightarrow \infty} x^{\prime}(t)=0$, and $\lim _{t \rightarrow \infty} x(t)=\ell$.

Remark 4. The following facts are employed in proving Lemma 5.

(i) There is a constant $\hat{C}=\hat{C}(\alpha)>0$ such that

$$
(u+v)^{1 / \alpha} \leq \hat{C}\left(u^{1 / \alpha}+v^{1 / \alpha}\right) \quad \text { for all } u, v \geq 0 .
$$

(ii) When (3) and (4) hold, we have the estimate

$$
\begin{aligned}
\int_{t}^{\infty} Q(s) & \left(\int_{s}^{\infty} Q(r)^{(1+\alpha) / \alpha} d r\right)^{1 / \alpha} d s \\
& \leq\left(\int_{t}^{\infty} Q(s)^{(1+\alpha) / \alpha} d s\right)\left\{\int_{t}^{\infty}\left(\int_{s}^{\infty} Q(r)^{(1+\alpha) / \alpha} d r\right)^{1 / \alpha} d s\right\}^{1 /(\alpha+1)} .
\end{aligned}
$$

Actually, since $\alpha /(\alpha+1)+1 /(\alpha+1)=1$, Hölder's inequality implies that

$$
\begin{aligned}
\int_{t}^{\infty} Q(s) & \left(\int_{s}^{\infty} Q(r)^{(1+\alpha) / \alpha} d r\right)^{1 / \alpha} d s \\
\leq & \left(\int_{t}^{\infty} Q(s)^{(1+\alpha) / \alpha} d s\right)^{\alpha /(1+\alpha)}\left\{\int_{t}^{\infty}\left(\int_{s}^{\infty} Q(r)^{(1+\alpha) / \alpha} d r\right)^{(\alpha+1) / \alpha} d s\right\}^{1 /(\alpha+1)} \\
= & \left(\int_{t}^{\infty} Q(s)^{(1+\alpha) / \alpha} d s\right)^{\alpha /(1+\alpha)} \\
& \times\left\{\int_{t}^{\infty}\left(\int_{s}^{\infty} Q(r)^{(1+\alpha) / \alpha} d r\right)\left(\int_{s}^{\infty} Q(r)^{(1+\alpha) / \alpha} d r\right)^{1 / \alpha} d s\right\}^{1 /(\alpha+1)} \\
\leq & \left(\int_{t}^{\infty} Q(s)^{(1+\alpha) / \alpha} d s\right)\left\{\int_{t}^{\infty}\left(\int_{s}^{\infty} Q(r)^{(1+\alpha) / \alpha} d r\right)^{1 / \alpha} d s\right\}^{1 /(\alpha+1)} .
\end{aligned}
$$


Proof of Lemma 5. We notice that a positive solution $x$ of equation (1) satisfies the conditions $\lim _{t \rightarrow \infty} x^{\prime}(t)=0$ and $\lim _{t \rightarrow \infty} x(t)=\ell$ if and only if it satisfies the integro-differential equation

$$
x(t)=\ell+\int_{t}^{\infty}\left\{Q(s) x(s)^{-\beta}-\beta \int_{s}^{\infty} Q(r) x(r)^{-\beta-1} x^{\prime}(r) d r\right\}^{(1 / \alpha) *} d s, \quad t \geq t_{0} .
$$

Here we have employed the definition of $Q(t)$ and integral by part, and we use the notation $c^{p *}=|c|^{p-1} c$ for $c \in \boldsymbol{R}$ and $p>0$. We try to find a positive solution of this equation. The proof is devided into several steps.

Step 1: We firstly construct a positive solution of (5) near $+\infty$ in a suitable function space by Schauder-Tychonoff's fixed point theorem ([8, Theorems 2.3.8 and 4.5.1]). Let $k, m>0$, and $T \geq t_{0}$ be numbers such that

$$
\begin{gathered}
\hat{C} \ell^{-\beta / \alpha} \leq m ; \\
\hat{C}^{2} \beta^{1 / \alpha} \ell^{-(\beta+1) / \alpha}\left[m^{1 / \alpha}+k^{1 / \alpha}\left\{\int_{T}^{\infty}\left(\int_{S}^{\infty} Q(r)^{(\alpha+1) / \alpha} d r\right)^{1 / \alpha} d s\right\}^{1 /(\alpha(\alpha+1))}\right] \leq k
\end{gathered}
$$

and

$$
\int_{T}^{\infty}\left[m Q(t)^{1 / \alpha}+k\left(\int_{t}^{\infty} Q(s)^{(\alpha+1) / \alpha} d s\right)^{1 / \alpha}\right] d t \leq \ell .
$$

Here $\hat{C}$ denotes the number introduced in (i) of Remark 4 . Consider the linear space $C^{1}[T, \infty)$ consisting of all continuously differentiable functions on $[T, \infty)$. We regard it as a Fréchet space equipped with the usual topology; that is, a sequence $\left\{x_{n}\right\} \subset C^{1}[T, \infty)$ converges to $x \in C^{1}[T, \infty)$ if and only if $\lim _{n \rightarrow \infty} x_{n}(t)=x(t)$ and $\lim _{n \rightarrow \infty} x_{n}^{\prime}(t)=x^{\prime}(t)$ uniformly on each compact subinterval in $[T, \infty)$. Let $X \subset C^{1}[T, \infty)$ be the closed non-empty subset of $C^{1}[T, \infty)$ consisting of all functions $x(t)$ satisfying inequalities

$$
\ell \leq x(t) \leq 2 \ell, \quad t \geq T
$$

and

$$
0 \leq-x^{\prime}(t) \leq m Q(t)^{1 / \alpha}+k\left(\int_{t}^{\infty} Q(s)^{(1+\alpha) / \alpha} d s\right)^{1 / \alpha}, \quad t \geq T .
$$

For $x \in X$ we define $\mathscr{F} x(t)$ by the right hand side of (5). Below we will show that $\mathscr{F}$ is a continuous mapping such that $\mathscr{F} X \subset X$ and $\mathscr{F} X$ is relatively compact. 
Let $x \in X$. We then have

$$
\begin{aligned}
& 0 \leq\left(\int_{t}^{\infty} Q(s) x(s)^{-\beta-1}\left[-x^{\prime}(s)\right] d s\right)^{1 / \alpha} \\
& \leq \ell^{-(\beta+1) / \alpha}\left[m \int_{t}^{\infty} Q(s)^{(\alpha+1) / \alpha} d s+k \int_{t}^{\infty} Q(s)\left(\int_{s}^{\infty} Q(r)^{(\alpha+1) / \alpha} d r\right)^{1 / \alpha} d s\right]^{1 / \alpha} \\
& \leq \hat{C} \ell^{-(\beta+1) / \alpha}\left[\left(m \int_{t}^{\infty} Q(s)^{(\alpha+1) / \alpha} d s\right)^{1 / \alpha}\right. \\
& \left.+\left\{k \int_{t}^{\infty} Q(s)\left(\int_{s}^{\infty} Q(r)^{(\alpha+1) / \alpha} d r\right)^{1 / \alpha} d s\right\}^{1 / \alpha}\right] \\
& \leq \hat{C} \ell^{-(\beta+1) / \alpha}\left[\left(m \int_{t}^{\infty} Q(s)^{(\alpha+1) / \alpha} d s\right)^{1 / \alpha}\right. \\
& \left.+\left(k \int_{t}^{\infty} Q(s)^{(\alpha+1) / \alpha} d s\right)^{1 / \alpha}\left\{\int_{t}^{\infty}\left(\int_{s}^{\infty} Q(r)^{(1+\alpha) / \alpha} d r\right)^{1 / \alpha} d s\right\}^{1 /(\alpha(\alpha+1))}\right] \\
& \leq \hat{C} \ell^{-(\beta+1) / \alpha}\left[m^{1 / \alpha}+k^{1 / \alpha}\left\{\int_{T}^{\infty}\left(\int_{s}^{\infty} Q(r)^{(1+\alpha) / \alpha} d r\right)^{1 / \alpha} d s\right\}^{1 /(\alpha(\alpha+1))}\right] \\
& \times\left(\int_{t}^{\infty} Q(s)^{(\alpha+1) / \alpha} d s\right)^{1 / \alpha} \\
& \leq \hat{C}^{-1} \beta^{-1 / \alpha} k\left(\int_{t}^{\infty} Q(s)^{(\alpha+1) / \alpha} d s\right)^{1 / \alpha} .
\end{aligned}
$$

Here we have used (ii) of Remark 4. It follows therefore that

$$
\begin{aligned}
0 & \leq-(\mathscr{F} x)^{\prime}(t) \leq \hat{C}\left\{Q(t)^{1 / \alpha} x(t)^{-\beta / \alpha}+\beta^{1 / \alpha}\left(\int_{t}^{\infty} Q(s) x(s)^{-\beta-1}\left[-x^{\prime}(s)\right] d s\right)^{1 / \alpha}\right\} \\
& \leq \hat{C} \ell^{-\beta / \alpha} Q(t)^{1 / \alpha}+k\left(\int_{t}^{\infty} Q(s)^{(\alpha+1) / \alpha} d s\right)^{1 / \alpha} \\
& \leq m Q(t)^{1 / \alpha}+k\left(\int_{t}^{\infty} Q(s)^{(\alpha+1) / \alpha} d s\right)^{1 / \alpha} .
\end{aligned}
$$

Similarly we have 


$$
\begin{aligned}
0 & \leq \mathscr{F} x(t)-\ell=-\int_{t}^{\infty}(\mathscr{F} x)^{\prime}(s) d s \\
& \leq \int_{t}^{\infty}\left[m Q(t)^{1 / \alpha}+k\left(\int_{t}^{\infty} Q(s)^{(\alpha+1) / \alpha} d s\right)^{1 / \alpha}\right] d t \leq \ell,
\end{aligned}
$$

implying that $\mathscr{F} x \in X$. Consequently we find that $\mathscr{F} X \subset X$.

To prove the continuity of $\mathscr{F}$, let a sequence $\left\{x_{n}\right\} \subset X$ converge to $x \in X$. We put for $x \in X$

$$
I[x](t)=\int_{t}^{\infty} Q(s) x(s)^{-\beta-1}\left[-x^{\prime}(s)\right] d s .
$$

As seen before we have by (6)

$$
0 \leq Q(s) x(s)^{-\beta-1}\left[-x^{\prime}(s)\right] \leq c_{1} Q(s)^{(\alpha+1) / \alpha}+c_{2} Q(s)\left(\int_{s}^{\infty} Q(r)^{(1+\alpha) / \alpha} d r\right)^{1 / \alpha}
$$

for some positive constants $c_{1}$ and $c_{2}$ not depending on $x$. Since the right hand side is integrable on $[T, \infty)$, the dominated convergence theorem implies that

$$
\begin{aligned}
& \left|I\left[x_{n}\right](t)-I[x](t)\right| \\
& \quad \leq \int_{T}^{\infty}\left|Q(s) x_{n}(s)^{-\beta-1}\left[-x_{n}^{\prime}(s)\right]-Q(s) x(s)^{-\beta-1}\left[-x^{\prime}(s)\right]\right| d s \rightarrow 0 \quad \text { as } n \rightarrow \infty .
\end{aligned}
$$

Hence we find that $\lim _{n \rightarrow \infty} I\left[x_{n}\right](t)=I[x](t)$ uniformly on $[T, \infty)$. Since

$$
-(\mathscr{F} x)^{\prime}(t)=\left\{Q(t) x(t)^{-\beta}+\beta I[x](t)\right\}^{1 / \alpha},
$$

we find that $\lim _{n \rightarrow \infty}\left(\mathscr{F} x_{n}\right)^{\prime}(t)=(\mathscr{F} x)^{\prime}(t)$ uniformly on each compact subinterval in $[T, \infty)$.

Next, we recall by the definition of $X$ that $\mathscr{F} x$ satisfies

$$
\left|(\mathscr{F} x)^{\prime}(t)\right| \leq m Q(t)^{1 / \alpha}+k\left(\int_{t}^{\infty} Q(s)^{(1+\alpha) / \alpha} d s\right)^{1 / \alpha}, \quad t \geq T .
$$

Notice that the function on the right hand side of this inequality is integrable on $[T, \infty)$. Since

$$
\left|\mathscr{F} x(t)-\mathscr{F} x_{n}(t)\right| \leq \int_{t}^{\infty}\left|(\mathscr{F} x)^{\prime}(s)-\left(\mathscr{F} x_{n}\right)^{\prime}(s)\right| d s
$$

and we have just shown that $\lim _{n \rightarrow \infty}\left(\mathscr{F} x_{n}\right)^{\prime}(t)=(\mathscr{F} x)^{\prime}(t)$, the dominated convergence theorem implies that $\lim _{n \rightarrow \infty} \mathscr{F} x_{n}(t)=\mathscr{F} x(t)$ uniformly on each compact subinterval in $[T, \infty)$. Hence $\mathscr{F}$ is continuous.

Finally, we show the relative compactness of the set $\mathscr{F} X$. It suffices, by Ascoli-Arzelà's theorem, to show that the set $\left\{(\mathscr{F} x)^{\prime}: x \in X\right\}$ is equicontinuous 
on each compact subinterval in $[T, \infty)$. Let $0<\alpha \leq 1$. Since each $\mathscr{F} x, x \in X$, is of class $C^{2}[T, \infty)$ and

$$
(\mathscr{F} x)^{\prime \prime}(t)=-\frac{1}{\alpha}\left\{Q(t) x(t)^{-\beta}+\beta I[x](t)\right\}^{(1-\alpha) / \alpha} q(t) x(t)^{-\beta},
$$

the set $\left\{(\mathscr{F} x)^{\prime \prime}: x \in X\right\}$ is uniformly bounded on each compact subinterval in $[T, \infty)$. Next let $\alpha>1$ and $t_{1}, t_{2} \in[T, \infty)$. Noting the inequality $\left|u^{1 / \alpha}-v^{1 / \alpha}\right| \leq|u-v|^{1 / \alpha}$ for $u, v \geq 0$, we have

$$
\begin{aligned}
\left|(\mathscr{F} x)^{\prime}\left(t_{1}\right)-(\mathscr{F} x)^{\prime}\left(t_{2}\right)\right| \leq & \mid\left\{Q\left(t_{1}\right) x\left(t_{1}\right)^{-\beta}-Q\left(t_{2}\right) x\left(t_{2}\right)^{-\beta}\right\} \\
& +\left.\beta\left\{I[x]\left(t_{1}\right)-I[x]\left(t_{2}\right)\right\}\right|^{1 / \alpha} \\
\leq & \left|Q\left(t_{1}\right) x\left(t_{1}\right)^{-\beta}-Q\left(t_{2}\right) x\left(t_{2}\right)^{-\beta}\right|^{1 / \alpha} \\
& +\beta^{1 / \alpha}\left|I[x]\left(t_{1}\right)-I[x]\left(t_{2}\right)\right|^{1 / \alpha} \\
= & \left|Q\left(t_{1}\right)\left\{x\left(t_{1}\right)^{-\beta}-x\left(t_{2}\right)^{-\beta}\right\}+\left\{Q\left(t_{1}\right)-Q\left(t_{2}\right)\right\} x\left(t_{2}\right)^{-\beta}\right|^{1 / \alpha} \\
& +\beta^{1 / \alpha}\left|\int_{t_{1}}^{t_{2}} Q(s) x(s)^{-\beta-1}\left[-x^{\prime}(s)\right] d s\right|^{1 / \alpha} .
\end{aligned}
$$

Since the integrand of the last integral is dominated by an integrable function on $[T, \infty)$ by $(7)$, the set $\left\{(\mathscr{F} x)^{\prime}: x \in X\right\}$ is equicontinuous on each compact subinterval of $[T, \infty)$.

From Schauder-Tychonoff's fixed point theorem there is a fixed point $x \in X$ of $\mathscr{F}: \mathscr{F} x=x$.

Step 2: We will show that $x^{\prime}(t)<0, t \geq T$, where $x$ is the solution of (5) just constructed above. To see this suppose the contrary that $x^{\prime}(\tilde{t})=0$ for some $\tilde{t} \geq T$. Since

$$
\left[-x^{\prime}(t)\right]^{\alpha}=Q(t) x(t)^{-\beta}+\beta I[x](t) \geq 0,
$$

we have $Q(\tilde{t})=I[x](\tilde{t})=0$. Therefore by the definition of $I[x](t)$ we find that $Q(t)\left[-x^{\prime}(t)\right] \equiv 0$ on $[\tilde{t}, \infty)$. It follows that

$$
Q(t)^{\alpha}\left[-x^{\prime}(t)\right]^{\alpha}=Q(t)^{1+\alpha} x(t)^{-\beta}+\beta Q(t)^{\alpha} I[x](t) \equiv 0, \quad t \geq \tilde{t} .
$$

Consequently, we have $Q(t)^{1+\alpha} x(t)^{-\beta} \equiv 0$, that is $Q(t) \equiv 0, t \geq \tilde{t}$. This contradicts the noncompactness of $\operatorname{supp} q$. Hence $x^{\prime}(t)<0$ on $[T, \infty)$.

Step 3: This is the final step. Since the exponent $-\beta$ of the nonlinear term of equation (1) is negative, and $x(t)$ is strictly decreasing on $[T, \infty)$, we can easily show that $x(t)$ can be extended to the left as a positive solution of 
equation (1) so that $x(t)$ exists on the whole interval $\left[t_{0}, \infty\right)$, and $x^{\prime}(t)<0$ on $\left[t_{0}, \infty\right)$. This completes the proof of Lemma 5 .

Proof of Theorem 1. By Lemma 5, we can find a sequence of functions $\left\{x_{n}\right\} \subset C^{1}\left[t_{0}, \infty\right)$ satisfying $x_{n}^{\prime}(t)<0 \quad$ on $\left[t_{0}, \infty\right), \quad \lim _{t \rightarrow \infty} x_{n}(t)=1 / n$, and $\lim _{t \rightarrow \infty} x_{n}^{\prime}(t)=0$, and

$$
\begin{aligned}
x_{n}(t) & =\frac{1}{n}+\int_{t}^{\infty}\left(\int_{s}^{\infty} q(r) x_{n}(r)^{-\beta} d r\right)^{(1 / \alpha) *} d s \\
& =\frac{1}{n}+\int_{t}^{\infty}\left\{Q(s) x_{n}(s)^{-\beta}+\beta \int_{s}^{\infty} Q(r) x_{n}(r)^{-\beta-1}\left[-x_{n}^{\prime}(r)\right] d r\right\}^{1 / \alpha} d s, \quad t \geq t_{0} .
\end{aligned}
$$

Below we show that the limit function of a suitable subsequence of $\left\{x_{n}\right\}$ gives rise to a desired positive solution of (1)-(2).

The decreasing nature of $x_{n}$ and (8) imply for $n \in N$ and $t \geq t_{0}$ that

$$
x_{n}(t) \geq x_{n}(t)^{-\beta / \alpha} \int_{t}^{\infty} Q(s)^{1 / \alpha} d s
$$

that is,

$$
x_{n}(t) \geq\left(\int_{t}^{\infty} Q(s)^{1 / \alpha} d s\right)^{\alpha /(\alpha+\beta)} .
$$

Thus from (8) again we obtain

$$
x_{n}(t) \leq 1+\int_{t}^{\infty}\left\{\int_{s}^{\infty}|q(r)|\left(\int_{r}^{\infty} Q(u)^{1 / \alpha} d u\right)^{-\alpha \beta /(\alpha+\beta)} d r\right\}^{1 / \alpha} d s
$$

Since

$$
-x_{n}^{\prime}(t)=\left(\int_{t}^{\infty} q(s) x_{n}(s)^{-\beta} d s\right)^{(1 / \alpha) *}
$$

by (9) we have

$$
\left|x_{n}^{\prime}(t)\right| \leq\left\{\int_{t}^{\infty}|q(s)|\left(\int_{s}^{\infty} Q(r)^{1 / \alpha} d r\right)^{-\alpha \beta /(\alpha+\beta)} d s\right\}^{1 / \alpha} .
$$

We find from (10) and (11) that the sequence $\left\{x_{n}\right\}$ is uniformly bounded and equicontinuous on $\left[t_{0}, \infty\right)$. Therefore Ascoli-Arzelà's theorem and a diagonal argument imply that a subsequence $\left\{x_{n_{i}}\right\} \subset\left\{x_{n}\right\}$ converges to a function $\tilde{x} \in C\left[t_{0}, \infty\right)$ uniformly on each compact subset in $\left[t_{0}, \infty\right)$. We notice by (9) that $\tilde{x}(t)>0, t \geq t_{0}$. Since $x_{n_{i}}$ satisfies

$$
x_{n_{i}}(t)=\frac{1}{n_{i}}+\int_{t}^{\infty}\left(\int_{s}^{\infty} q(r) x_{n_{i}}(r)^{-\beta} d r\right)^{1 / \alpha} d s
$$


we have

$$
\tilde{x}(t)=\int_{t}^{\infty}\left(\int_{s}^{\infty} q(r) \tilde{x}(r)^{-\beta} d r\right)^{1 / \alpha} d s,
$$

by letting $n_{i} \rightarrow \infty$. Here we have used the dominated convergence theorem. This formula shows obviously that $\tilde{x}$ is a positive solution of (1)-(2). The proof is completed.

Proof of Theorem 2. Let $\left\{x_{n}\right\}$ be the sequence introduced in the proof of Theorem 1. As before, we will show that $\left\{x_{n}\right\}$ contains a subsequence converging to a positive decaying solution by a method different from that of Theorem 1. To this end we establish for $\left\{x_{n}\right\}$ the following estimates for $n \in N$ and $t \geq t_{0}$ :

$$
\begin{gathered}
x_{n}(t)^{(\alpha+\beta) /(\alpha+1)} \leq n^{-(\alpha+\beta) /(\alpha+1)}+\frac{\alpha+\beta}{\alpha+1}\left(\frac{\alpha+1}{\alpha(1-\beta)}\right)^{1 /(\alpha+1)} \int_{t}^{\infty} \hat{q}(s)^{1 /(\alpha+1)} d s \\
{\left[-x_{n}^{\prime}(t)\right]^{\alpha+1} \leq \frac{\alpha+1}{\alpha(1-\beta)} \hat{q}(t) x_{n}(t)^{1-\beta}}
\end{gathered}
$$

and

$$
\left[-x_{n}^{\prime}(t)\right]^{\alpha} \geq \beta \int_{t}^{\infty} Q(s)^{(\alpha+1) / \alpha} x_{n}(s)^{-\beta-1-\beta / \alpha} d s
$$

In fact, rewriting equation (1) as

$$
\alpha\left|x_{n}^{\prime}\right|^{\alpha-1} x_{n}^{\prime \prime}=q(t) x_{n}^{-\beta}, \quad t \geq t_{0},
$$

and multiplying both sides by $-x_{n}^{\prime}(>0)$, we have

$$
-\left(\frac{\alpha}{\alpha+1}\left|x_{n}^{\prime}\right|^{\alpha+1}\right)^{\prime}=-q(t) x_{n}^{-\beta} x_{n}^{\prime} .
$$

In view of the decreasing nature of $\hat{q}$, by integrating this formula on $[t, \infty)$ we have

$$
\begin{aligned}
\frac{\alpha}{\alpha+1}\left|x_{n}^{\prime}(t)\right|^{\alpha+1} & \leq \int_{t}^{\infty}|q(s)| x_{n}(s)^{-\beta}\left[-x_{n}^{\prime}(s)\right] d s \\
& \leq \hat{q}(t) \int_{t}^{\infty} x_{n}(s)^{-\beta}\left[-x_{n}^{\prime}(s)\right] d s \\
& =\frac{\hat{q}(t)}{1-\beta} x_{n}(t)^{1-\beta} .
\end{aligned}
$$

Thus (13) is proved. Since (13) is rewritten as 


$$
-\left(x_{n}(t)^{(\alpha+\beta) /(\alpha+1)}\right)^{\prime} \leq \frac{\alpha+\beta}{\alpha+1}\left(\frac{\alpha+1}{\alpha(1-\beta)}\right)^{1 /(\alpha+1)} \hat{q}(s)^{1 /(\alpha+1)},
$$

an integration on $[t, \infty)$ of this inequality gives (12). Finally, since

$$
\left[-x_{n}^{\prime}(t)\right]^{\alpha}=Q(t) x_{n}(t)^{-\beta}+\beta \int_{t}^{\infty} Q(s) x_{n}(s)^{-\beta-1}\left[-x_{n}^{\prime}(s)\right] d s,
$$

by (8), we have

$$
-x_{n}^{\prime}(t) \geq Q(t)^{1 / \alpha} x_{n}(t)^{-\beta / \alpha}
$$

and

$$
\left[-x_{n}^{\prime}(t)\right]^{\alpha} \geq \beta \int_{t}^{\infty} Q(s) x_{n}(s)^{-\beta-1}\left[-x_{n}^{\prime}(s)\right] d s .
$$

Combining these inequalities we can obtain (14).

From (12) and (13) we find that $\left\{x_{n}\right\}$ and $\left\{x_{n}^{\prime}\right\}$ are uniformly bounded on $\left[t_{0}, \infty\right)$. Moreover (9), (13), (14) and equation (15) imply that $\left\{x_{n}^{\prime \prime}\right\}$ is uniformly bounded on each compact subinterval in $\left[t_{0}, \infty\right)$. Hence, by Ascoli-Arzelà's theorem and the diagonal argument it follows that $\left\{x_{n}\right\}$ contains a subsequence $\left\{x_{n_{i}}\right\}$ satisfying $\lim _{n_{i} \rightarrow \infty} x_{n_{i}}(t)=\tilde{x}(t)$ and $\lim _{n_{i} \rightarrow \infty} x_{n_{i}}^{\prime}(t)=\tilde{x}^{\prime}(t)$ for some $\tilde{x} \in C^{1}\left[t_{0}, \infty\right)$ uniformly on each compact subinterval in $\left[t_{0}, \infty\right)$. Letting $n_{i} \rightarrow \infty$ in the formulas

$$
\left[-x_{n_{i}}^{\prime}(t)\right]^{\alpha}=\left[-x_{n_{i}}^{\prime}\left(t_{0}\right)\right]^{\alpha}-\int_{t_{0}}^{t} q(s) x_{n_{i}}(s)^{-\beta} d s, \quad t \geq t_{0},
$$

and

$$
x_{n_{i}}(t)^{(\alpha+\beta) /(\alpha+1)} \leq n_{i}^{-(\alpha+\beta) /(\alpha+1)}+\frac{\alpha+\beta}{\alpha+1}\left(\frac{\alpha+1}{\alpha(1-\beta)}\right)^{1 /(\alpha+1)} \int_{t}^{\infty} \hat{q}(s)^{1 /(\alpha+1)} d s,
$$

we find that $\tilde{x}$ is a desired positive solution of (1)-(2). (Note that the positivity of $\tilde{x}$ is guaranteed by (9).) The proof is finished.

Proof of Theorem 3. Let $\left\{x_{n}\right\}$ be the sequence introduced in the proof of Theorem 1. As before, we will show that $\left\{x_{n}\right\}$ contains a subsequence converging to a positive decaying solution. We establish for $\left\{x_{n}\right\}$ the following estimates for $n \in N$ and $t \geq t_{0}$ :

$$
\begin{gathered}
x_{n}(t)^{(\alpha+\beta) /(\alpha+\delta)} \leq c_{1} n^{-(\alpha+\beta) /(\alpha+\delta)}+c_{2} \int_{t}^{\infty}\left(\int_{s}^{\infty}|q(r)|^{1 /(1-\delta)} d r\right)^{(1-\delta) /(\alpha+\delta)} d s \\
0<-x_{n}^{\prime}(t) \leq c_{3}\left(\int_{t}^{\infty}|q(s)|^{1 /(1-\delta)} d s\right)^{(1-\delta) /(\alpha+\delta)} x_{n}(t)^{(\delta-\beta) /(\alpha+\delta)} .
\end{gathered}
$$


Here $c_{1}, c_{2}$ and $c_{3}$ are positive constants independent of $n$ and $t$. To see (16), we multiply equation $(1)$ by $\left(-x_{n}^{\prime}\right)^{\delta}$ :

$$
-\left(\frac{\alpha}{\alpha+\delta}\left(-x_{n}^{\prime}\right)^{\alpha+\delta}\right)^{\prime}=q(t) x_{n}^{-\beta}\left(-x_{n}^{\prime}\right)^{\delta} .
$$

We integrate this on $[t, \infty)$ with noting the fact that $x_{n}^{\prime}(t)<0$ and $(1-\delta)+\delta=1$ :

$$
\begin{aligned}
\frac{\alpha}{\alpha+\delta}\left[-x_{n}^{\prime}(t)\right]^{\alpha+\delta} & =\int_{t}^{\infty} q(s) x_{n}(s)^{-\beta}\left[-x_{n}^{\prime}(s)\right]^{\delta} d s \\
& \leq \int_{t}^{\infty}|q(s)| x_{n}(s)^{-\beta}\left[-x_{n}^{\prime}(s)\right]^{\delta} d s \\
& \leq\left(\int_{t}^{\infty}|q(s)|^{1 /(1-\delta)} d s\right)^{1-\delta}\left(\int_{t}^{\infty} x_{n}(s)^{-\beta / \delta}\left[-x^{\prime}(s)\right] d s\right)^{\delta} \\
& \leq\left(\frac{\delta}{\delta-\beta}\right)^{\delta}\left(\int_{t}^{\infty}|q(s)|^{1 /(1-\delta)} d s\right)^{1-\delta} x_{n}(t)^{\delta-\beta} .
\end{aligned}
$$

This gives (17). The inequality (16) follows from (17) by an integration. As in the proof of Theorem 2, we can show that $\left\{x_{n}\right\}$ contains a subsequence converging to a desired positive solution of problem (1)-(2). This completes the proof.

Example 1. Let us consider problem (1)-(2) under the condition that $q$ satisfies

$$
c_{1} t^{-v_{1}} \leq q(t) \leq c_{2} t^{-v_{2}} \quad \text { near }+\infty
$$

for some constants $c_{1}, c_{2}>0$ and $v_{1}, v_{2} \in \boldsymbol{R}$. Theorem 1 shows that, if

$$
\alpha+1<v_{2} \leq v_{1}<\frac{\alpha}{\beta}\left(v_{2}-\alpha-1\right)+v_{2},
$$

then problem (1)-(2) has a positive decaying solution.

In fact, the typical equation

$$
\left(\left|x^{\prime}\right|^{\alpha-1} x^{\prime}\right)^{\prime}=t^{-v} x^{-\beta}, \quad v>\alpha+1, t \geq 1,
$$

has a positive decaying solution $x_{0}$ explicitly given by

$$
x_{0}(t)=c_{0} t^{-\mu}, \quad t \geq 1,
$$

where

$$
\mu=\frac{v-\alpha-1}{\alpha+\beta} \quad \text { and } \quad c_{0}^{\alpha+\beta}=\frac{1}{\alpha \mu^{\alpha}(\mu+1)}=\frac{(\alpha+\beta)^{\alpha+1}}{\alpha(v-\alpha-1)^{\alpha}(v+\beta-1)} .
$$


Example 2. Let $0<\beta<1$ and $v>\alpha+1$. Then the problem

$$
\begin{gathered}
\left(\left|x^{\prime}\right|^{\alpha-1} x^{\prime}\right)^{\prime}=t^{-v-1}\{v(1+\sin t)-t \cos t\} x^{-\beta}, \quad t \geq 1 \\
\lim _{t \rightarrow \infty} x(t)=0
\end{gathered}
$$

has a positive solution. To show this by Corollary 4, we observe that the coefficient function $q(t)$ of this equation is $O\left(t^{-v}\right)$, and $\int_{t}^{\infty} q(t) d t=$ $t^{-v}(1+\sin t) \geq 0$. Note that the coefficient function $q(t)$ oscillates, and hence known existence results in [4] are not applicable.

\section{A remark for uniqueness of positive decaying solutions}

As is stated in Example 1 in Section 2, the typical equation

$$
\left(\left|x^{\prime}\right|^{\alpha-1} x^{\prime}\right)^{\prime}=t^{-v} x^{-\beta}, \quad v>\alpha+1, t \geq 1,
$$

has a positive decaying solution $x=x_{0}$ explicitly given by (18) and (19). If $\alpha=1$, we proved that $x_{0}$ is the only positive decaying solution of (20); see [7] for the details. We here show that, for this typical equation, such a uniqueness theorem is still valid.

Theorem 6. Equation (20) has a unique positive decaying solution explicitly given by (18) and (19).

This theorem is proved via asymptotic theory of planar dynamical systems. We need the following lemmas:

Lemma 7. Let $x$ be a positive decaying solution of (20). Then we have

$$
0<\liminf _{t \rightarrow \infty} \frac{x(t)}{x_{0}(t)} \leq \limsup _{t \rightarrow \infty} \frac{x(t)}{x_{0}(t)}<\infty,
$$

and

$$
0<\liminf _{t \rightarrow \infty} \frac{-x^{\prime}(t)}{-x_{0}^{\prime}(t)} \leq \limsup _{t \rightarrow \infty} \frac{-x^{\prime}(t)}{-x_{0}^{\prime}(t)}<\infty,
$$

where $x_{0}$ is given by (18) and (19).

Proof. Computing as in the proof of (9), we can prove the validity of the first inequality in (21). Once we get an estimate of $x(t)$ from below, we can get an estimate of $x(t)$ from above by equation (1). Since

$$
-x^{\prime}(t)=\left(\int_{t}^{\infty} s^{-v} x(s)^{-\beta} d s\right)^{1 / \alpha}
$$


we see from the just verified inequality that

$$
\begin{aligned}
-x^{\prime}(t) & \leq c_{1}\left(\int_{t}^{\infty} s^{-v}\left(s^{-(v-\alpha-1) /(\alpha+\beta)}\right)^{-\beta} d s\right)^{1 / \alpha} \\
& =c_{2} t^{-(v+\beta-1) /(\alpha+\beta)} \equiv \widetilde{c_{2}}\left[-x_{0}^{\prime}(t)\right], \quad t \geq 1
\end{aligned}
$$

for some constants $c_{1}, c_{2}, \widetilde{c_{2}}>0$. This proves the validity of the last inequality in (22). The first inequality in (22) can be shown similarly. This completes the proof.

Lemma 8. Let

$$
t=e^{s} \quad \text { and } \quad x / x_{0}(t)=z, \quad(\dot{z}-\mu z)^{\alpha *}=w,
$$

where $\cdot=d / d s$ and $\mu=(v-\alpha-1) /(\alpha+1)$. Then, equation (20) is transformed into the dynamical system

$$
\left\{\begin{array}{l}
\dot{z}=\mu z+w^{(1 / \alpha) *} \\
\dot{w}=\alpha(\mu+1) w+\alpha \mu^{\alpha}(\mu+1) z^{-\beta} .
\end{array}\right.
$$

Proof of Theorem 6. The proof is done by contradiction. Let $x$ be a positive decaying solution of (20) which does not coincide with $x_{0}$. Let us perform the change of variables introduced in Lemma 8 . We denote $(z(s), w(s))=\varphi(s)$ below. The only critical point of the dynamical system (23) lying in the region $z>0$ is $P_{0}=\left(1,-\mu^{\alpha}\right)$. Notice that the particular solution $x_{0}$ of equation (20) corresponds to the critical point $P_{0}$.

Let $J(z, w)$ be the Jacobi matrix of the right hand side of system (23). It is easily seen that

$$
J\left(1,-\mu^{\alpha}\right)=\left(\begin{array}{cc}
\mu & \alpha^{-1} \mu^{1-\alpha} \\
-\alpha \beta \mu^{\alpha}(\mu+1) & \alpha(\mu+1)
\end{array}\right) .
$$

Therefore the real parts of the eigenvalues of $J\left(1,-\mu^{\alpha}\right)$ are all positive. We can find an open ball $B$ centered at $P_{0}$ with a sufficiently small radius such that $\varphi(s) \in B^{c}$ for $s \geq 0$; see [5, Section 26]. By Lemma 7 we can find a compact set $K$ lying in the region $z>0$ such that $\varphi(s) \in K$ for $s \geq 0$. We may suppose that $K$ contains $B$ entirely. Hence, Poincare-Bendixson's theorem asserts that system (23) has a periodic solution contained in $K$.

On the other hand, since

$$
\frac{\partial}{\partial z}\left(\mu z+w^{(1 / \alpha) *}\right)+\frac{\partial}{\partial w}\left\{\alpha(\mu+1) w+\alpha \mu^{\alpha}(\mu+1) z^{-\beta}\right\} \equiv \mu+\alpha(\mu+1)>0,
$$

Bendixson's theorem implies that system (23) does not have periodic solutions. This is an obvious contradiction. This completes the proof. 
Acknowledgement. The authors would like to thank the referee for his or her careful reading of the manuscript and many useful comments.

\title{
References
}

[1] Ad'yutov, M. M. and Klokov, Yu. A. and Mikhailov, A. P., Some problems for an ordinary differential equations arising in gas dynamics, Differential Equations, 26 (1990), 803-805.

[2] Callegari, A. J. and Nachman, A., Some singular, nonlinear differential equations arising in boundary layer theory, J. Math. Anal. Appl., 64 (1978), 96-105.

[ 3 ] Kusano, T. and Swanson, C. A., Asymptotic theory of singular semilinear elliptic equations, Canad. Math. Bull., 27 (1984), 223-232.

[4] Kusano, T. and Tanigawa, T., Positive solutions to a class of second order differential equations with singular nonlinearities, Appl. Anal., 69 (1998), 315-331.

[5] Pontriagin, L. S., A Course in Ordinary Differential Equations, Hindustan Publishing Corporation (India), Delhi, 1967.

[6] Usami, H., On positive decaying solutions of singular Emden-Fowler type equations, Nonlinear Anal., 16 (1991), 795-803.

[7] Usami, H., Asymptotic behavior of positive solutions of singular Emden-Fowler type equations, J. Math. Soc. Japan, 46 (1994), 195-211.

[8] Smart, D. R., Fixed Point Theorems, Cambridge University Press, London, 1974.

\author{
nuna adreso: \\ Misako Motai \\ Kanzaki Seimei High School \\ Kanzaki, Saga 842-0012 \\ Japan \\ Hiroyuki Usami \\ Department of Mathematics \\ Graduate School of Science \\ Hiroshima University \\ Higashi-Hiroshima 739-8521 \\ Japan \\ E-mail: usami@mis.hiroshima-u.ac.jp
}

(Ricevita la 26-an de oktobro, 2006)

(Reviziita la 27-an de aprilo, 2007) 